\title{
Cellular photoablation to control postoperative fibrosis in a rabbit model of filtration surgery
}

\author{
Salvatore Grisanti, Michael Diestelhorst, Klaus Heimann, Günter Krieglstein
}

\begin{abstract}
Aim-To evaluate the feasibility of cellular photoablation using fluorescence generated photoreaction products as a method to control postoperative fibrosis.

Methods-The fluorescent probe, 2',7'bis-(2-carboxyethyl)-5-(and-6)-carboxyfluorescein, acetoxymethyl ester (BCECF-AM) is a cell membrane permeable compound rendered membrane impermeable and fluorescent upon cleavage by intracellular esterases. Rabbits (ChB$B: C H ; n=20)$ received a unilateral subconjunctival injection of BCECF-AM (40, 70, 80 , or $100 \mu \mathrm{g}) 30$ minutes before surgery followed by intraoperative illumination with diffuse blue light (450-490 $\mathrm{nm}$; $\left.51.9 \times 10^{3} \mathrm{~cd} / \mathrm{m}^{2}\right)$ for 10 minutes. Controls received either the probe or illumination. Antifibrotic efficacy was established by clinical response and histological examination. Clinical response was assessed by comparing intraocular pressure (IOP) between the treated experimental eye and the fellow eye, which served as control. Success was defined by $>\mathbf{2 0} \%$ difference in IOP.
\end{abstract}

Results-IOP was significantly decreased in all groups within 4 days postoperatively. In control groups IOP rose within 10 days to normal levels. This was similar in the group receiving $40 \mu \mathrm{g}$ of BCECF-AM. In the other groups (subconjunctival injection of 70-100 $\mu \mathrm{g}$ BCECF-AM) IOP was significantly $(p<0.02)$ decreased for $2-3$ weeks. Clinical and histological examination revealed no toxic damage to adjacent tissues.

Conclusions-Cellular photoablation in contrast with chemotherapeutic agents acts on cells that have incorporated BCECF-AM and have been exposed to light at the appropriate wavelength. Though safety and reliability demand further studies this method might be an useful therapeutic approach to control postoperative fibrosis in humans undergoing filtration surgery.

(Br f Ophthalmol 1999;83:1353-1359)

Trabeculectomy is the most frequently applied surgical method to reduce intraocular pressure in patients with glaucoma. The wound healing process within the filtration area, however, is a cause of surgery failure in $30 \%$ of the cases. ${ }^{1}$ Characteristically fibroblasts from Tenon's capsule and episclera lead to a fibroproliferative response involving and closing the created fistula. ${ }^{2-12}$ To increase the success rates of filtering surgery several substances, such as corticosteroids $^{13-15}$ and antimetabolites (mitomycin-C and 5-fluorouracil) ${ }^{4}{ }^{16-34}$ have been used as antifibrotic therapy. Despite their positive effect on filtering surgery success rates, diffusion into adjacent ocular tissues also leads to toxic effects on other than the targeted cells. $^{35-37}$ Photodynamic therapy might be an alternative way to solve this problem. ${ }^{98}$ Photosensitisers can be used as mediators of light induced cell toxicity. They seem to act via the formation of reactive oxygen intermediates and free radicals. ${ }^{39-42}$ Selective activation of the photosensitiser by light application at the appropriate wavelength limits the drug effect on the targeted area. Several photosensitisers are currently under investigation in ophthalmology. ${ }^{43-47}$ Cellular photoablation can be mediated by BCECF-AM (2',7'-bis(2-carboxyethyl)-5-(and-6)-carboxyfluorescein, acetoxymethyl ester). The photoablative effect of this carboxyfluorescein derivate has been shown before ${ }^{40} 47$ and could be confirmed in our previously conducted in vitro experiments. BCECF-AM is a cell membrane permeable compound rendered membrane impermeable and fluorescent upon cleavage by intracellular esterases (Fig 1A). Exposure of cells that have incorporated BCECF-AM to light at the appropriate wavelength leads to cellular photoablation (Fig 1B, C).

The objective of this pilot study was to investigate the impact of cellular photoablation mediated by BCECF-AM on fibrosis after filtration surgery in a rabbit model.

\section{Methods}

\section{ANIMALS AND GROUPS}

All experiments were performed with chinchilla bastard rabbits (ChBB: $\mathrm{CH}$ ), 3-6 months old and weighing $1.5-2.5 \mathrm{~kg}$. Animals were obtained from Thomae (Biberach) and maintained in a common room of the vivarium at the university eye clinic of Cologne where an overhead fluorescent light provided 12 hour cycles of light and dark. Surgery and clinical 

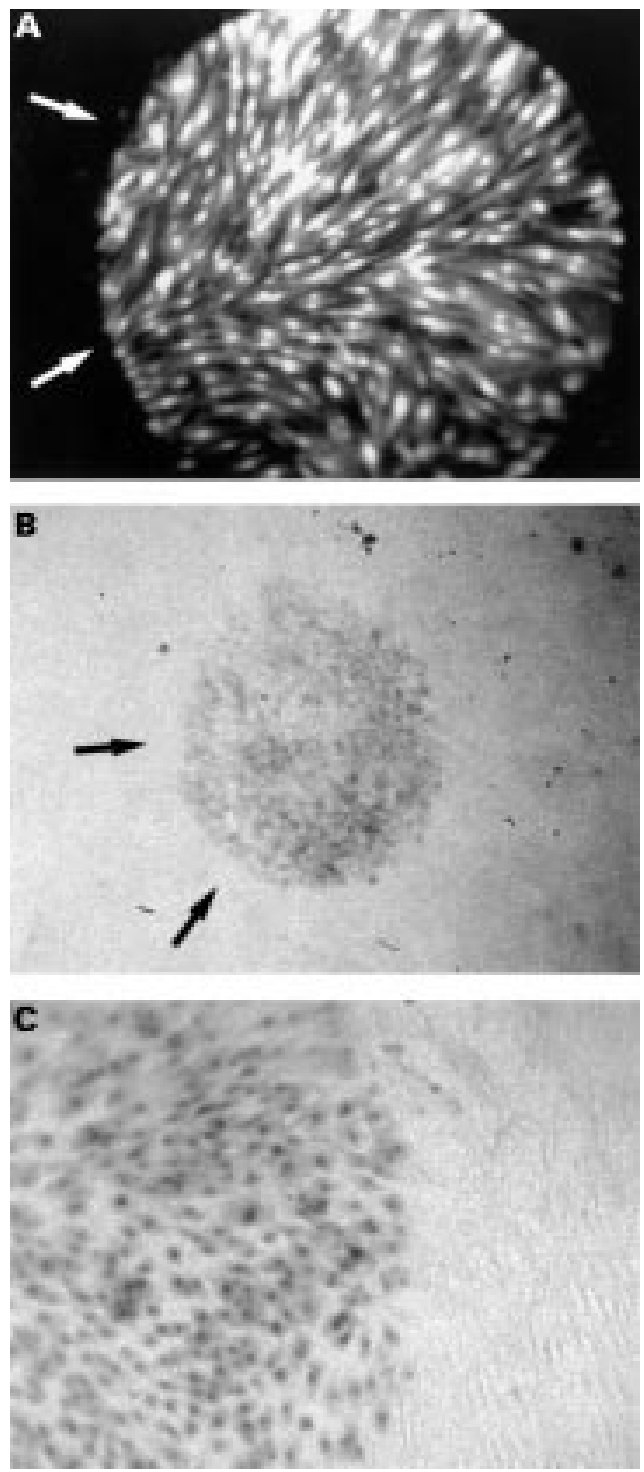

Figure 1 (A) Fluorescence photomicrograph of cultured human Tenon's capsule fibroblasts loaded with BCECF-AM. Cells within the circular area (arrows) are excited by diffuse blue light (450-490 nm) and fuoresce. (B) Phase contrast photomicrograph of cultured human Tenon's capsule

fibroblasts previously targeted by diffuse blue light (450-490 $\left.\mathrm{nm}, 1.68 \times 10^{2} \mathrm{~mW} / \mathrm{m}^{2}\right)$. Cells within the targeted circular area (arrows) incorporated trypan blue indicating cellular damage. (C) Magnification of (B) disclosing the targeted cell bodies which have incorporated trypan blue.

examinations were performed under anaesthesia induced by intramuscular injections of 1.5 $\mathrm{ml}$ ketamine (Ketanest, Parke Davis, Berlin, Germany) and $0.7 \mathrm{ml}$ xylazine (Rompun, Bayer, Leverkusen, Germany). Before enucleations of treated eyes animals were killed by lateral ear vein injection of $1 \mathrm{ml} \mathrm{T}-61$ (Hoechst, Frankfurt, Germany). All experimental proce-

Table 1 Schedule of treatment of the right eye of 20 rabbits

\begin{tabular}{llll}
\hline Groups & Function & No & Procedure \\
\hline Group A & control & 3 & Surgery + illumination, no BCECF-AM \\
Group B & experimental & 3 & Surgery + illumination + 40 $\mu \mathrm{g}$ BCECF-AM \\
Group C & experimental & 3 & Surgery + illumination + 80 $\mu \mathrm{g}$ BCECF-AM \\
Group D & control & 3 & Surgery + 80 $\mu \mathrm{g}$ BCECF-AM, no illumination \\
Group E & experimental & 3 & Surgery + illumination + 70 $\mu \mathrm{g}$ BCECF-AM \\
Group F & experimental & 3 & Surgery + illumination + 100 $\mu \mathrm{g}$ BCECF-AM \\
& control & 1 & $80 \mu \mathrm{g}$ BCECF-AM, illumination, no surgery \\
& control & 1 & $80 \mu \mathrm{g}$ BCECF-AM, no illumination, no surgery \\
\hline
\end{tabular}

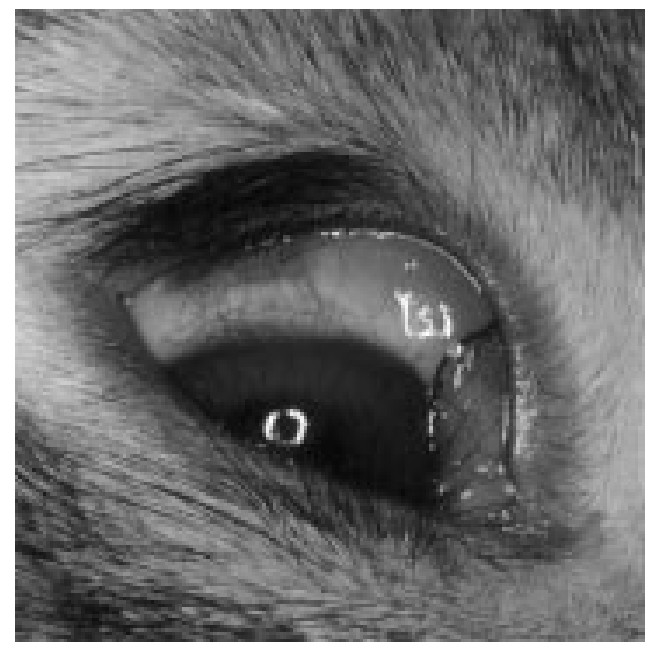

Figure 2 Aspect of the anterior segment of a rabbit eye after a subconjunctival injection of a BCECF-AM solution (100 $\mu l$ ) displaying a subconjunctival bleb filled with the yellowish solution.

dures conformed to the ARVO resolution on the use of animals in research and to institutional guidelines.

Surgical procedure was performed on 18 rabbits subdivided into the following six groups: group A and D served as controls; group $A(n=3)$ received no photosensitiser, but intraoperative illumination with diffuse blue light (10 minutes); group $\mathrm{D}(\mathrm{n}=3)$ received 30 minutes before surgery a subconjunctival injection of the photosensitiser $(80 \mu \mathrm{g})$, but no intraoperative illumination; groups $\mathrm{B}, \mathrm{C}, \mathrm{E}$, and $F(n=3$ per group) received before surgery different concentrations of the photosensitiser $(40-100 \mu \mathrm{g})$ and intraoperative illumination with diffuse blue light (10 minutes). Two animals served as controls without surgery. Both animals were given a subconjunctival injection of photosensitiser $(80 \mu \mathrm{g})$. Thirty minutes later one of these animals received a complete illumination of the anterior part of the eye for 10 minutes. The other animal received no illumination (Table 1). In all groups the fellow eye served as additional control.

PHOTOSENSITISER AND PHOTOACTIVATION

The fluorescent probe, 2',7'-bis-(2-carboxyethyl)-5-(and-6)-carboxyfluorescein, acetoxymethyl ester (BCECF-AM) was provided by Sigma-Aldrich (Steinheim) and diluted in balanced salt solution containing DMSO to experimental concentration. Animals received 30 minutes before surgery a unilateral (right eye) subconjunctival injection $(100 \mu \mathrm{l})$ of a BCECF-AM $(40-100 \mu \mathrm{g})$ solution in the superior quadrant (Fig 2). After photosensitiser injection rabbits remained in unchanged lighting conditions. Illumination with diffuse blue light $\left(450-490 \mathrm{~nm} ; 51.9 \times 10^{3} \mathrm{~cd} / \mathrm{m}^{2}, 10 \mathrm{~min}-\right.$ utes) was performed after peritomy and removal of superfluous, non-incorporated BCECF-AM by BSS, before filtration surgery. Photoactivating light was delivered by a portable slit lamp (Zeiss) equipped with a blue filter fixed at a distance of $10 \mathrm{~cm}$ from the target. The light was focused onto an area of 5.0 

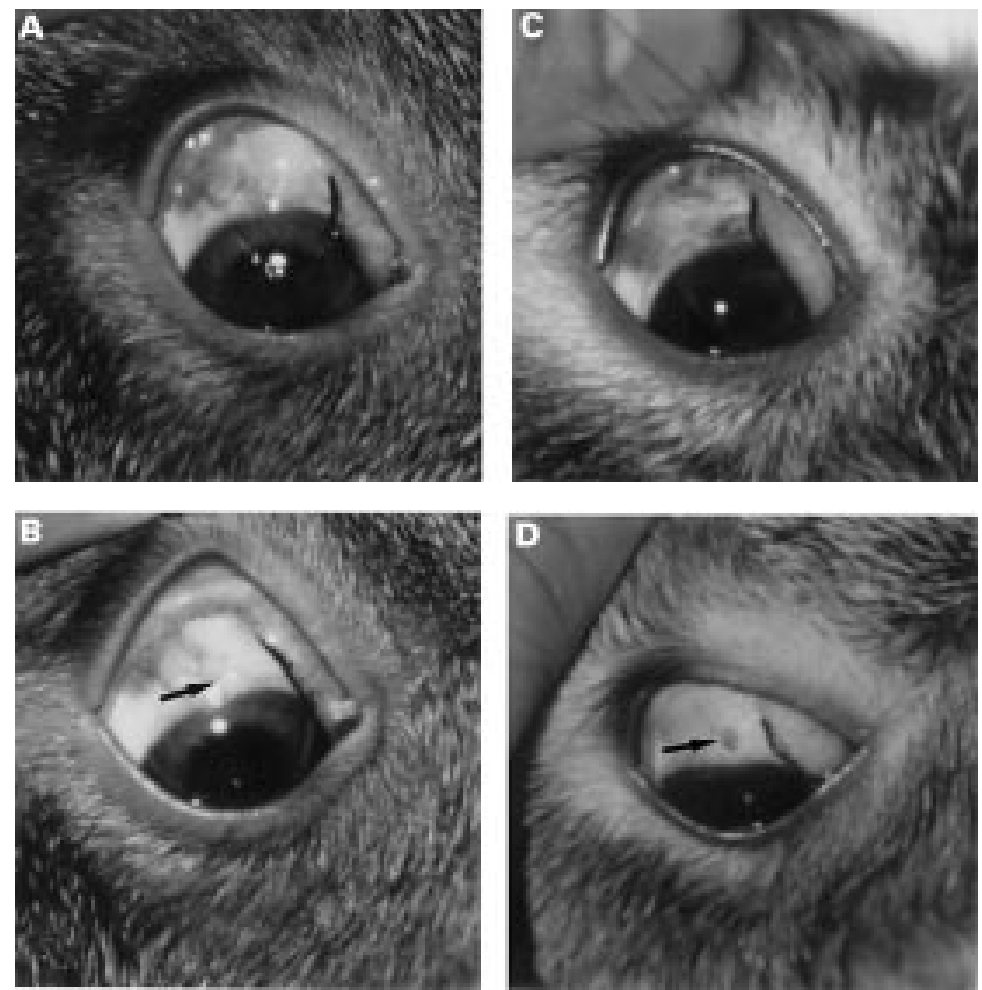

Figure 3 (A) Rabbit from control group D 6 days after surgery. The anterior segment displays a mild inflammation. Encapsulated, non-functional filtration bleb is expressed. IOP in this eye had already increased to normal levels. (B) Same animal as in (A) 10 days after surgery. Sclerostomy area is closed by scar tissue (arrow). (C) Rabbit from experimental group $F 6$ days after surgery. Anterior segment displays a mild inflammatory response. (D) The same animal as in (C) 10 days after surgery. Sclerostomy site is covered only partially by fibrotic tissue (arrow). The IOP in this eye was significantly $(p<0.02)$ reduced.

$\mathrm{mm}$ diameter (ø) comprising conjunctiva and episcleral tissue and sparing the cornea that was partly covered by the reverted conjunctival peritomy flap. The illuminated area encompassed the consequently produced sclerostomy (ø $1.5 \mathrm{~mm}$ ). Since the illuminated area was well focused, no mask was used to minimise light absorption to adjacent tissues.

\section{SURGICAL PROCEDURE}

The surgical procedure requiring 5-10 minutes was performed with the aid of a surgical microscope on previously anaesthetised animals. Additionally, local anaesthesia with
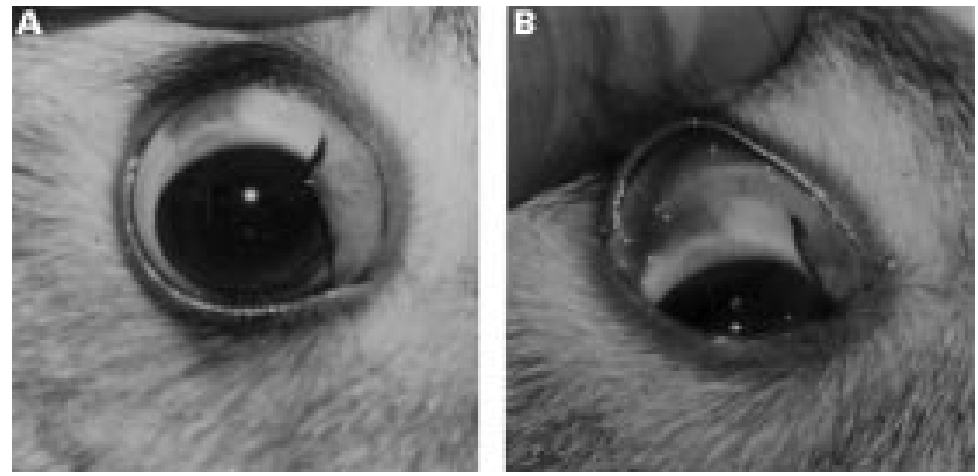

Figure 4 (A) Anterior segment of a control eye 10 days after subconjunctival injection of $B C E C F-A M(80 \mu \mathrm{g})$. This eye received neither surgery nor illumination with diffuse blue light. (B) Aspect of the anterior segment of a control eye 10 days after a subconjunctival injection of BCECF-AM injection ( $80 \mu \mathrm{g}$ ), and consequent (after 30 minutes) illumination of the anterior segment with diffuse blue light for 10 minutes. No surgery was performed on this eye. oxybuprocaine drops (Novesine $0.4 \%$, Ciba Vision) was applied. After placement of a lid speculum, a peritomy at $3.0-4.0 \mathrm{~mm}$ from the limbus was created in the superior quadrant of the right eye. To prevent intraocular entry of the fluorescent probe, filtration surgery was preceded by removal of non-incorporated BCECF-AM. Illumination of the surgical area was performed before filtration surgery. The entry into the anterior chamber was created with a trephine (ø $1.5 \mathrm{~mm}$ ) posterior to the limbal insertion of the conjunctiva. After the excision of the trephined block of tissue, a mid-peripheral iridectomy was performed. Tenon's capsule and conjunctiva were closed with a single 8-0 Vicryl suture. Postoperatively the eyes were treated with an antibiotic for 5 consecutive days. No steroids were applied.

\section{CLINICAL EXAMINATION}

Clinical examination was performed in order to evaluate both the general appearance of the treated eyes and to measure the intraocular pressure (IOP) of experimental and control eyes. On designated days, rabbits were anaesthetised and their eyes examined. In order to measure the IOP, the eyes were anaesthetised with proxymetacaine (proparacaine). Tonometry was performed with an applanation tonometer (Tono-Pen XL, Mentor, USA) before experimentation and postoperatively. To exclude interindividual and cyclic variations IOP was compared between the manipulated experimental eye and the non-manipulated fellow eye. The measurements were performed as triplicates. The difference in measured IOP was expressed as right eye/left eye ratio. Preoperative IOP ratios (R/L) ranged from 0.85 to 1.2. Success was defined by $>20 \%$ difference in IOP reflected as IOP ratio $<0.8$. A two tailed Student's $t$ test was performed and significance assumed to exist if $\mathrm{p}<0.05$.

\section{HISTOLOGICAL EXAMINATION}

On designated days eyes were enucleated and immediately fixed in $10 \%$ buffered formaldehyde for at least 24 hours. Consequently eyes were examined with a dissecting microscope (Zeiss) and a ring in the sagittal axis comprising the relevant area excised. Tissue samples were dehydrated and embedded in paraffin, 5 $\mu \mathrm{m}$ serial sections cut, rehydrated, stained with haematoxylin and eosin, and cover slipped.

\section{PHOTOGRAPHS}

Photographs were produced with a $35 \mathrm{~mm}$ camera system (Nikon). Micrographs of sections were taken with a $35 \mathrm{~mm}$ camera system attached to a Zeiss research microscope.

\section{Results}

CLINICAL APPEARANCE AND INTRAOCULAR

PRESSURE

The fate of the experimental and control eyes was evaluated clinically daily within the first week and twice a week thereafter.

Inflammatory response in surgical eyes such as conjunctival hyperaemia was mild in all groups and lasted for 1 week (Fig 3). There was no remarkable difference between injected 


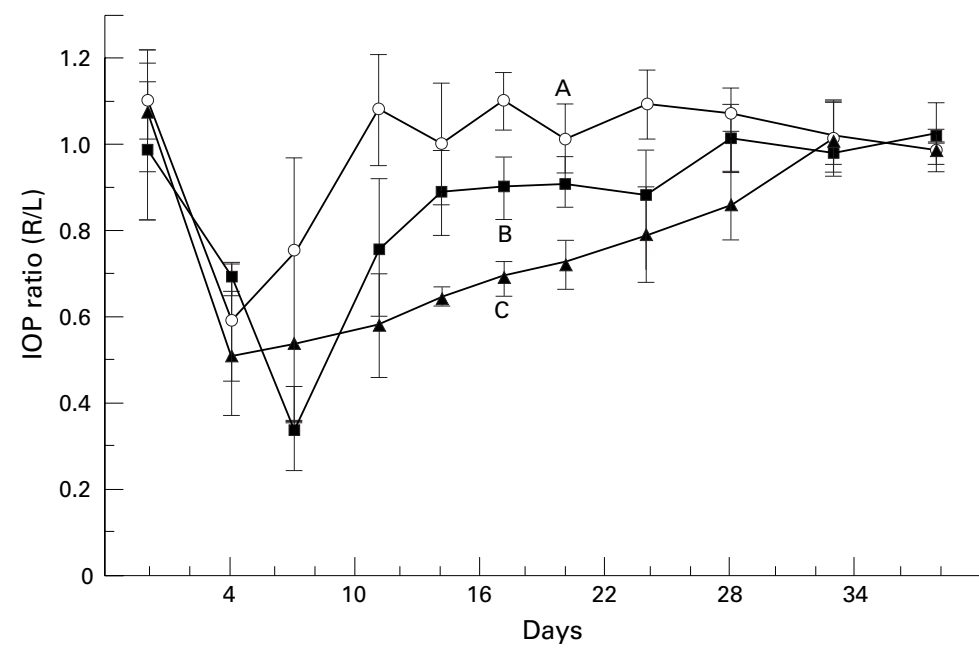

Figure 5 Intraocular pressure (IOP) measured as IOP ratios between experimental right and left eye preoperatively on surgery day (0) and postoperatively at distinct time points. Group $A$ (O received no photosensitiser but intraoperative illumination with diffuse blue light (450-490 nm). Group $B$ (汭 received $40 \mu \mathrm{g}$ and group $C$ received $80 \mu \mathrm{g}$ of $B C E C F-A M(\mathbf{\Delta})$ followed by intraoperative illumination for 10 minutes.

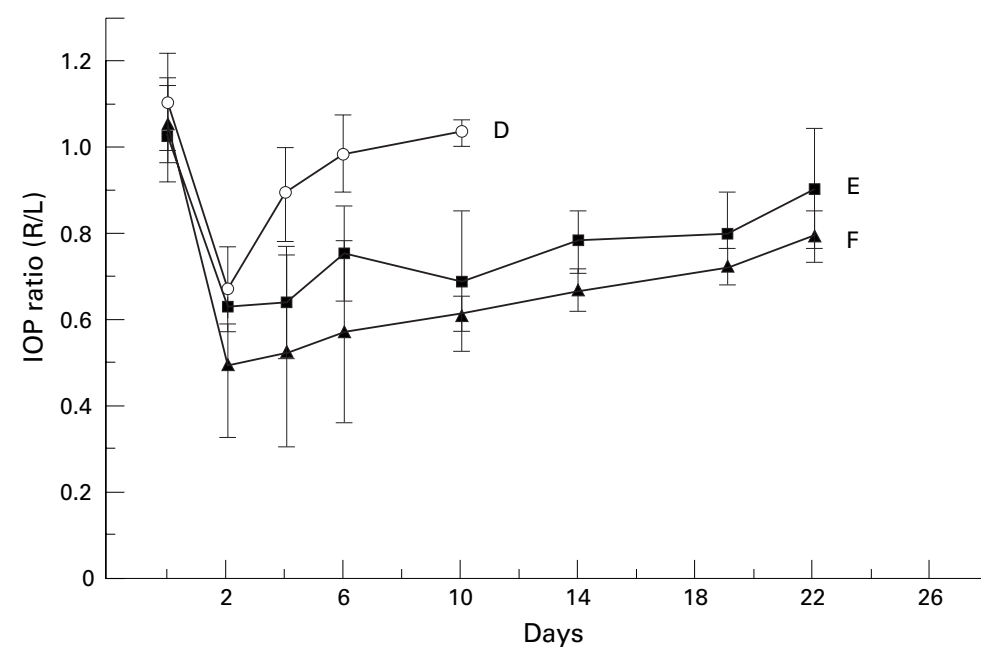

Figure 6 Intraocular pressure (IOP) measured as IOP ratios between experimental right and left eye preoperatively on surgery day (0) and postoperatively at distinct time points. Group $D$ (O received $80 \mu g B C E C F-A M$ subconjunctivally but no intraoperative

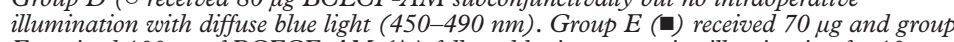
$F$ received $100 \mu \mathrm{g}$ of $B C E C F-A M(\mathbf{\Delta})$ followed by intraoperative illumination for 10 minutes.
Wound healing could be clinically observed as closure of the filtration area by ingrowing fibrotic tissue. This process was more prominent in the groups without photodynamic therapy (Fig 3). After a delay of 1 week it also progressed in the treated groups.

Intraocular pressure was measured as the mean of triplicates on designated days. Preoperative intraocular pressure ranged between 10-18 mm Hg. Preoperative IOP ratios (R/L) ranged from 0.85 to 1.2 and were drastically reduced in all groups within the second postoperative day displaying a mean value of 0.6 (Figs 5 and 6). In group A receiving intraoperative illumination without photosensitiser IOP ratios increased to preoperative values within 1 week. Group B treated with $40 \mu \mathrm{g}$ BCECF-AM showed a slight delay, but no significant difference from group A. Group C receiving $80 \mu \mathrm{g}$ BCECF-AM preoperatively retained a significant $(p<0.02)$ IOP decrease within 3 weeks postoperatively but consequently acquired preoperative values (Fig 5). To exclude an antifibrotic effect by the probe itself in the second set of experiments (Fig 6) Animals in group D received a subconjunctival injection of photosensitiser $(80 \mu \mathrm{g})$, but no intraoperative illumination. Similar to group A filtering surgery failure occurred within 1 week and IOP ratios increased to preoperative values. A reduced dose of BCECF-AM $(70 \mu \mathrm{g})$ was still effective and prolonged the surgical effect in group $\mathrm{E}$ for 2 weeks. To examine if a higher concentration could have a better impact on filtering surgery survival, animals in group $\mathrm{F}$ received a subconjunctival injection $100 \mu \mathrm{g}$ BCECF-AM. Though IOP was significantly $(p<0.02)$ decreased until day 14 , it increased thereafter and failure was assessed after the third week.

HISTOLOGICAL APPEARANCE

All rabbits in groups A-C were sacrificed on the 38th postoperative day and the treated eyes enucleated for histological examination. In the second set of experiments (groups D-F) treated eyes were enucleated on designated days. The tissues were stained with haematoxylin and eosin for light microscopy.

In the first set of experiments eyes were enucleated (38th postoperative day) when both control and experimental groups had already reached preoperative IOP ratios. Though fibrotic tissue ingrowth at the filtering area of group C appeared hypocellular compared with groups $\mathrm{A}$ and $\mathrm{B}$, the sclerostomy site was closed in all groups and obviously responsible for IOP increase.

In the second set of experiments control eyes receiving subconjunctival injection of BCECF-AM without intraoperative illumination were enucleated on postoperative day 10, when surgery had failed and IOP was increased. Histological analysis of these tissues disclosed a sclerostomy site that was obstructed by hypercellular fibrotic tissue (Fig 7A). To evaluate the wound healing process in an experimental eye with functioning filtering area, a rabbit in group $\mathrm{F}$ was sacrificed on postoperative day 10 , when the IOP ratio was 

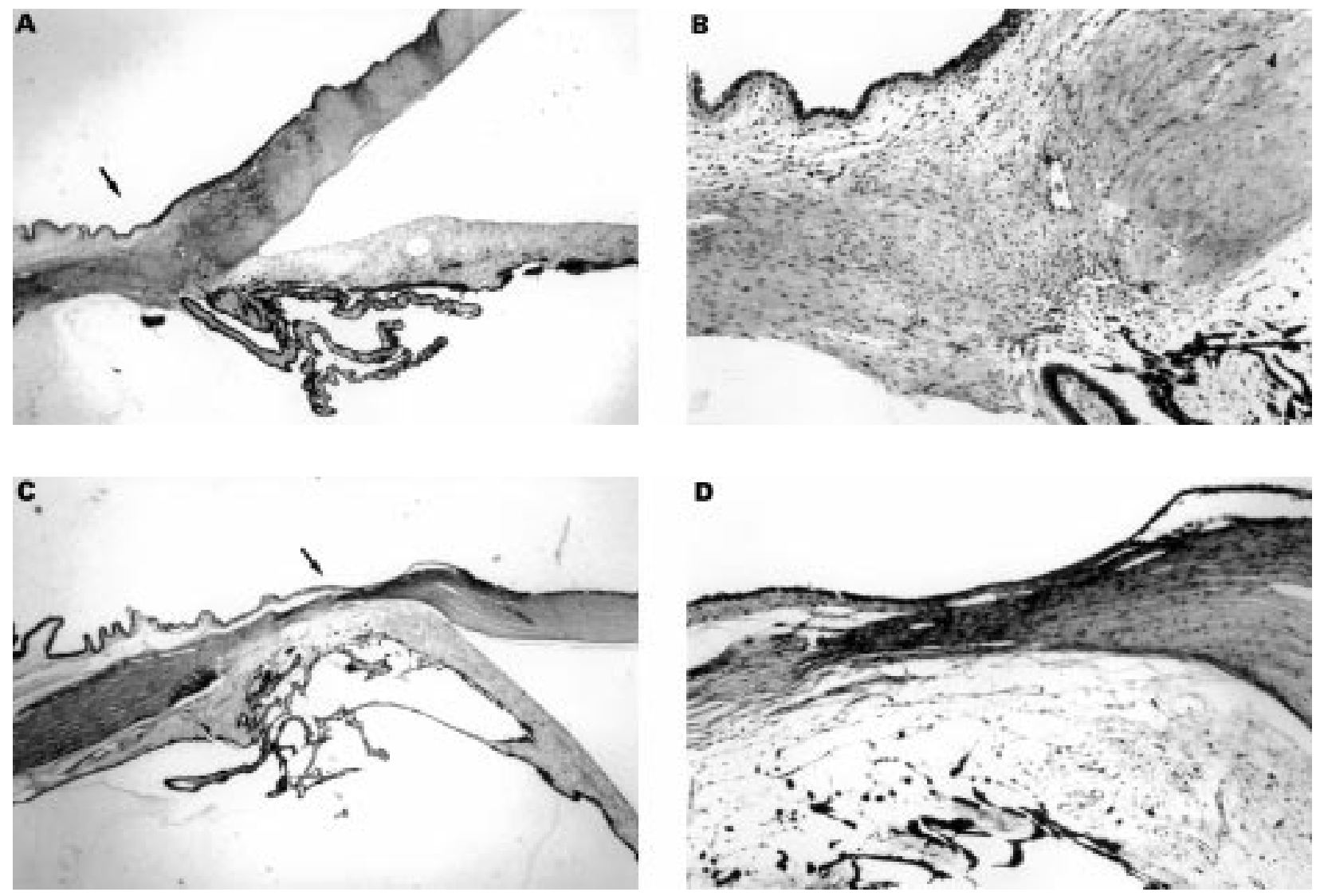

Figure 7 (A) Histological aspect of the surgical area 10 days after surgery in a rabbit from group D. The sclerostomy site (arrow) is infiltrated by hypercellular fibrotic tissue. IOP in this eye had already risen to normal levels. (B) Magnification of the area in (A). (C) Histological appearance of the surgical area 10 days after surgery in a rabbit from group $F$. The sclerostomy site (arrow) is infiltrated by a hypocellular lamellar scar tissue. The IOP ratio in this animal was still significantly $(p<0.02)$ reduced to 0.6 . (D) Magnification of the area in $(C)$.

0.6. Histological profiles revealed a mild lamellar ingrowth of hypocellular fibrotic tissue within the filtering zone (Fig 7B). Though a complete open fistula could not be found, it is conceivable that this thin lamella might allow filtration of aqueous humour.

\section{Discussion}

Photodynamic therapy is an alternative method for the treatment of localised pathologies such as skin cancers. ${ }^{43}$ Specific activation of pertinent drugs at the targeted area should avoid systemic side effects. Photodynamic therapy has also been evaluated for some distinct ophthalmological diseases such as ocular tumours, ${ }^{20}{ }^{44}$ choroidal $^{45}$ and corneal ${ }^{46}$ neovascularisation, proliferative vitreoretinal disorders, ${ }^{47}$ and postoperative fibrosis in glaucoma surgery. ${ }^{9}$ A recent study has investigated the feasibility of photodynamic therapy in a rabbit model of filtration surgery. Using ethyl etiopurin, a photosensitiser traditionally delivered by intravenous injection, Hill et al showed that subconjunctival delivery could have an impact on filtering bleb survival. ${ }^{38}$

The aim of this pilot study was to evaluate photodynamic therapy for antifibrosis using BCECF-AM. This carboxyfluorescein derivative is a cell membrane permeable compound rendered membrane impermeable and fluorescent upon cleavage by intracellular esterases. ${ }^{48-50}$ Exposure of cells that have incorporated BCECF-AM to light at the appropri- ate wavelength leads to cellular photoablation (Fig 1). The light induced cytotoxic ability of BCECF-AM has been shown before in in vitro studies. ${ }^{40} 47$

In the present pilot study we tested BCECF-AM in a rabbit model of filtration surgery. Because of the aggressive wound healing response in rabbits, this animal model is believed to be equivalent to high risk eyes in humans and surgical failure results within 1 week. The use of antimetabolites, such as 5-fluorouracil and mitomycin C, will prolong bleb survival. However, the effect is not permanent and depending on drug concentration it will subside within a few weeks. ${ }^{321}$

Our experiments show that filtration surgery success can be prolonged when cellular photoablation is applied. As demonstrated by groups $\mathrm{A}$ and D wound closure occurs and IOP will increase within 1 week when one of the components (photosensitiser, illumination with blue light) necessary for photoablation is missing. This time for surgical failure is similar to previous reports and demonstrates that nonactivated photosensitiser or illumination has no detectable impact on wound closure.

In group B receiving $40 \mu \mathrm{g}$ of BCECF-AM photoablative therapy seemed to be ineffective, since surgical failure occurred, such as in control groups A and D, within 10 days (Figs 5 and 6). Using BCECF-AM at a concentration of $70-100 \mu \mathrm{g}$ filtration surgery success could be prolonged for about 3 weeks. This was 
expressed both in lowered IOP levels (Figs 5 and 6) and reduced fibrosis at the sclerostomy site (Figs 5 and 7 ).

Though some authors claim the creation of filtration blebs as evidence of successful surgery, in our hands blebs were not always associated with reduced IOP. This is probably due to encapsulation. Such a case is demonstrated in Figure 3A, which displays a rabbit eye from group $\mathrm{D}$. Though a filtering bleb is still present on the 10th postoperative day, IOP had already risen to normal levels (Fig 6). Filtration blebs were, therefore, not evaluated as markers of succesful treatment.

BCECF-AM will be incorporated by all cells including proliferating and non-proliferating cells. Both clinical and light microscopic examination revealed no detectable damage in adjacent non-illuminated tissues. However, ultrastructure should be evaluated in further studies. BCECF-AM is like other drugs and may diffuse into other tissues. However, because illumination is achieved with diffuse light, which can activate the dye only at the surface of the tissue irradiated, it is not thought that decreased IOP results from a photoablative effect on other tissues-for example, the ciliary body. Though it might have been expected, subconjunctival injection of BCECF-AM $(80 \mu \mathrm{g})$ followed by illumination did not cause a conjunctival defect. A possible explanation for this result might be an insufficient transmission of the diffuse blue light through the intact conjunctiva and/or the insufficient penetration of the probe into the superficial areas. In evaluating in vitro studies cellular photoablation could be only detected in cells that had incorporated BCECF-AM and were exposed to light at the appropriate wavelength (450-490 $\mathrm{nm}$ ) irradiated by a microscope. Since daylight includes the exciting wavelength, we evaluated the effect of daylight on scleral fibroblasts that had incorporated BCECF-AM and could exclude a lethal effect (results not shown). This result might be because of the lesser light intensity than that from a microscope. Furthermore, in the animal model and clinical practice, the upper lid will protect the injected area.

A more intense light exposure will occur during surgery because of the operating microscope. In group D, however, this circumstance did not seem to have an effect on the wound healing process (Fig 6). In practice, a photoablative effect induced by the operating microscope would be beneficial when limited to the filtration area, but critical in adjacent tissues such as the cornea. Though the results are promising in this pilot study, further studies are necessary to optimise the effect by adjusting drug concentration and irradiation time.

Cellular photoablation might be an alternative to chemotherapeutic agents as a therapeutic approach to control postoperative fibrosis in glaucomatous eyes. Safety and reliability need to be studied in the future.

This study was supported by the Deutsche Forschungsgemeinschaft (HE 840/7-1); Retinovit-Foundation.

Proprietary interest: None.
This work was presented in part at the Association of Research in Vision and Ophthalmology Annual Meeting, May 1998, Ft Lauderdale, Florida, USA.

1 Maumenee AE. External filtering operations for glaucoma: the mechanisms of function and failure. Trans Am Ophthalmol Soc 1960;58:319-28.

2 Addicks EM, Quigley HA, Green WR, et al. Histologic characteristics of filtering blebs in glaucomatous eyes. Arch Ophthalmol 1983;101:795-8.

3 Costa VP, Spaeth GL, Eiferman RA, et al. Wound healing modulation in glaucoma filtration surgery. Ophthalmic Surg 1993;24:152-70.

4 Heuer DK, Parrish II RK, Gressel MG, et al. 5-Fluorouracil and glaucoma filtering surgery. Intermediate follow-up of a pilot-study. Ophthalmology 1986;93:1537-46.

5 Hitchings RA, Grierson I. Clinicopathological correlation in eyes with failed fistulizing surgery. Trans Ophthalmol Soc UK 1983;103:84-88.

6 Jampel HD, McGuigan LJB, Dunkelberger GR, et al. Cellular proliferation after experimental glaucoma filtration surgery. Arch Ophthalmol 1988;106:89-94.

7 Joseph JP, Miller MH, Hitchings RA. Wound healing as a barrier to successul filtration surgery. Eye 1988;2:S113-23.

8 Skuta GL, Parrish II RK. Wound healing in glaucoma filtering surgery. Surv Ophthalmol 1987;32:149-70

9 Smyth RJ, Nguyen K, Ahn SS, et al. The effects of photophrin on human Tenon's capsule fibroblasts in vitro. f Ocular Pharmacol 1993; 9:171-8.

10 Teng CC, Chi HH, Katzin HM. Histology and mechanism of filtering operations. Am f Ophthalmol 1959;47:16-34.

11 Von Sengbusch G, Couwenbergs C, Kühner J, et al. Fluorogenic substrate turnover in single living cell. Histochemstry 1976;8:341-50

12 Weinreb RN. Wound healing in filtration surgery. Curr Opin Ophthalmol 1994;13:8-25.

13 Roth SM, Spaeth GL, Starita RJ, et al. The effect of postoperative corticosteroids on trabeculectomy and the clinical course of glaucoma: five-year follow-up study. Ophthalmic Surg 1991;22:724-9.

14 Starita RJ, Fellmann RL, Spaeth GL, et al. Short- and longterm effects of postoperative corticosteroids on trabeculectomy. Ophthalmology 1985;92:938-46.

15 Sugar HS. Clinical effect of corticosteroids on conjuctival filtering blebs: a case report. Am $\mathcal{f}$ Ophthalmol 1965;59: $854-60$

16 Bansal RK, Gupta A. 5-Fluorouracil in trabeculectomy for patients under the age of 40 years. Ophthalmic Surg 23:278-80.

17 Bergstrom TJ, Wilkinson S, Skuta GL, et al. The effect of subconjuctival mitomycin C on glaucoma filtration surgery in rabbits. Arch Ophthalmol 1991;109:1725-30.

18 Chen CW, Huang HT, Bair JS, et al. Trabeculectomy with simultaneus topical application of mitomycin C in refractory glaucoma. F Ocular Pharmacol 1990;6:175-82.

19 Heuer DK, Parrish II RK, Gresel MG, et al. 5-Fluorouracil and glaucoma filtration surgery. II. A pilot study. Ophthalmology 1984;91:384-94.

20 Jampel HD, Jabs DA, Quigley HA. Trabeculectomy with 5 -fluorouracil for adult inflammatory glaucoma. $\mathrm{Am} f$ Ophthalmol 1990;109:168-73.

21 Khaw PT, Doyle JW, Sherwood MB, et al. Effects of intraoperative 5 -fluorouracil or mitomycin $\mathrm{C}$ on glaucoma filtration surgery in the rabbit. Ophthalmology 1993;100: 367-72.

22 Kitazawa Y, Kawase K, Matsushita H, et al. Trabeculectomy with mitomycin. A comparative study with fluorouracil. Arch Ophthalmol 1991;109:1693-8.

23 Lee DA, Hersh P, Kersten D, et al. Complications of subconjuctival 5-fluorouracil following glaucoma filtering surgery. Ophthalmic Surg 1987;18:187-90

24 Lee DA, Lee, TC, Cortes AE, et al. The effect of 5 -fluorouracil and cytarabine on human fibroblasts from Tenon's capsule. Invest Ophthalmol Vis Sci 1990;31:213644.

25 Lee DA, Shapourifar-Tehrani S, Kitada S. The effect of 5-fluorouracil and cytarabine on human fibroblasts from Tenon's capsule. Invest Ophthalmol Vis Sci 1990;31:184855.

26 Liang LL, Epstein DL. Comparison of mitomycin C and 5 -fluorouracil on filtration surgery success in rabbit eyes. $\mathcal{F}$ Glaucoma 1992;1:87-93.

27 Mermoud A, Salmon JF, Murray ADN. Trabeculectomy with mitomycin $\mathrm{C}$ for refractory glaucoma in blacks. $A m \mathcal{F}$ Ophthalmol 1993;116:72-8.

28 Palmer SS. Mitomycin as adjunct chemotherapy with trabeculectomy. Ophthalmology 1991;98:317-21.

29 Pasquale LR, Thibault D, Dorman-Pease ME, et al. Effect of topical mitomycin C on glaucoma filtering surgery in monkeys. Ophthalmology 1992;99:14-18.

30 Patitsas CJ, Rockwood EJ, Meisler DM, et al. Glaucoma filtering surgery with post-operative 5-fluorouracil in patients with intraocular inflammatory disease. Ophthalmology 1992;99:594-9.

31 Rockwood EJ, Parrish II RK, Heuer DK, et al. Glaucoma filtering surgery with 5-fluorouracil. Ophthalmology 1987; 94:1071-8.

32 Tahery MM, Lee DA. Review: pharmacologic control of wound healing in glaucoma filtering surgery. $\mathcal{F}$ Ocular Pharmacol 1989;5:155-79.

33 The Fluorouracil Filtering Surgery Study Group. Threeyear follow-up of the Fluorouracil Filtering Surgery Study. Am f Ophthalmol 1993;115:82-92. 
34 Wilson MR, Lee DA, Baker RS, et al. The effects of topical mitomycin on glaucoma filtration surgery in rabbits. $\mathcal{F ~ O c u}$ mitomycin on glaucoma filtr.

35 Costa VP, Wilson RP, Moster MR, et al. Hypotony maculopathy following the use of topical mitomycin C in glaucoma filtration surgery. Ophthalmic Surg 1993;24:38994.

36 Shields MB, Scroggs MW, Sloop CM, et al. Clinical and histopathologic observations concerning hypotony after trabeculectomy with adjunctive mitomycin C. Am f Ophthalmol 1993;116:673-83.

37 Skuta GL, Beeson CC, Higginbotham EJ, et al. Intraoperative mitomycin versus postoperative 5-fluorouracil in high risk glaucoma filtering surgery. Ophthalmology 1992;99:438-44.

38 Hill RA, Crean DH, Doiron DR, et al. Photodynamic therapy for antifibrosis in a rabbit model of filtration surgery. Ophthalmic Surg Lasers 1997;28:574-81.

39 Cohan CS, Hadley RD, Kater SB. Zap axotomy: localized fluorescent exitation of single dye-filled neurons induces growth by selective axotomy. Brain Res 1983;270:93-101.

40 Dulon D, Zajic G, Schacht J. Photo-induced irreversible shortening and swelling of isolated cochlear outer hair cells. shortening and swelling of isolated coct

41 Miller JP, Selverstone AJ. Rapid killing of single neurons by intracellularly injected dye. Science 1979;206:702-4.

42 Moreno G, Lutz M, Bessis M. Partial cell irradiation by ultraviolet and visible light: conventional and laser sources. Int Rev Exp Pathol 1969;7:99-136.
43 Dougherty TJ, Marcus SL. Photodynamic therapy. Eur $\mathcal{F}$ Cancer 1992;28:1734-42.

44 Weissgold DJ, Hu LK, Gragoudas ES, et al. Photodynamic therapy (PDT) of pigmented choroidal melanoma via a trans-scleral approach. Invest Ophthalmol Vis Sci 1996;37: (S) 123. (Abstract no 588.)

45 Moshfeghi DM, Peyman GA, Khoobehi B, et al. Photodynamic occlusion of retinal vessels using tin ethyl etiopurin SnET2): an efficacy study. Invest Ophthalmol Vis Sci 1995; 36:(S) 115. (Abstract no 564.)

46 Cox KW, Shepperd JD, Lattanzio FA, et al. Photodynamic therapy of corneal neovascularization using topical dihematoporphyrin ester. Invest

47 Dacheux R, Guidry C. Cellular photoablation as a therapy to control proliferative vitreoretinopathy: in vitro studies. Invest Ophthalmol Vis Sci 1995;36:(S)751. (Abstract no 3476.)

48 Grimes PA, Stone RA, Laties AM, et al. Carboxyfluorescein. A probe of the blood-ocular barriers with lower membrane permeability than fluorescein. Arch Ophthalmol 1982;100: permea

49 Hofmann J, Sernetz M. A kinetic study on the enzymatic hydrolysis of fluoresceindiacetate and fluorescein-di-betaD-galactopyranoside. Analyt Biochem 1983;131:180-6.

50 Rotman B, Papermaster B. Membrane properties of living mamalian cells as studied by enzymatic hydrolysis of fluorogenic esters. Proc Natl Acad Sci USA 1966;55:134-41. 